\title{
Response to 'Gene-by-environment experiments: a new approach to finding the missing heritability' by van IJzendoorn et al.
}

\section{Duncan Thomas}

In response to my Review (Gene-environment-wide association studies: emerging approaches. Nature Reviews Genetics 11, 259-272 (2010)) $)^{1}$, van IJzendoorn et al. (Gene-by-environment experiments: a new approach to finding the missing heritability. Nature Rev. Genet. 18 Nov 2011 (doi:10.1038/nrg2764-c1) $)^{2}$ suggest that the problems caused by misclassifying the environment when assessing gene by environment $(\mathrm{G} \times \mathrm{E})$ interactions can largely be avoided when exposure is assigned at random, as in a clinical trial. I made similar arguments, with examples, in the section of my paper on 'Experimental validation of $\mathrm{G} \times \mathrm{E}$ interactions' (REF. 1). Although many hazardous environmental factors are not amenable to random assignment for obvious ethical reasons, pharmacogenetics is an example of one area where it has been possible to study genetic determinants of response to drugs within randomized controlled trials. Furthermore, it is expected that $\mathrm{G} \times \mathrm{E}$ interactions seen with new drugs could be larger than those seen with ubiquitous environmental exposures, for which natural selection might have eliminated the susceptibility alleles ${ }^{3}$. To further develop this theme, I would like to offer a few other examples to illustrate potential extensions of this idea.

One of the examples I cited in my Review ${ }^{1}$ was a randomized crossover trial of the effects of challenge with diesel exhaust particles or ragweed allergen on a panel of immunological markers and their modification by the glutathione-S-transferase genes GSTM1 and
GSTP1 (REF. 4). We have subsequently shown how the estimates of genetic main effects and $\mathrm{G} \times \mathrm{E}$ interactions that are derived from such studies can be used as priors to inform the analysis of a much larger epidemiological cohort study ${ }^{5}$.

Another example illustrates the use of experimental techniques to analyse nonrandomized epidemiological data. In the Children's Health Study ${ }^{6}$, we are conducting toxicological assays of the biological effectiveness of particulate pollution samples that were collected at the homes of study participants and using them to challenge standard cell lines and lines in which GSTM1 has been knocked out (GSTM1 is a strong candidate modifier of air pollution sensitivity). Here, the exposure is applied in a controlled manner across genotypes to derive indices of 'biologically effective dose' that can be used in the analysis of exposure-response relationships and $\mathrm{G} \times \mathrm{E}$ interactions in the epidemiological study.

This approach could also be extended to perform assays on cell cultures that are derived from actual study subjects to assess interactions of exposure with background genetic variation in free-living human populations. Such an approach is being applied to study colony survival following radiation, ataxia telangiectasia mutated (ATM) expression and kinase activity and checkpoint activation in cell cultures derived from participants of the WECARE study of second breast cancers following radiotherapy ${ }^{7,8}$. The resulting estimates of biological activity can serve as prior covariates in a hierarchical model for studying the interaction between radiation dose and specific variants in the ATM gene in epidemiological study.

In summary, there are numerous opportunities beyond the analysis of genetic variation within randomized exposure studies (for example, clinical trials) to exploit experimental methods to improve the study of $\mathrm{G} \times \mathrm{E}$ interactions. There are currently only very limited ontologies available for the joint effects of genes and environmental factors that could be used to inform epidemiological analyses ${ }^{9}$. Closer collaborations of epidemiologists with toxicologists and experimental biologists will help move this field forward.

The author is at the Department of Preventive
Medicine, University of Southern California,
2001 N. Soto Street, C-202F, MC 9234,
Los Angeles, California 90089-9234, USA.
e-mail: dthomas@usc.edu
doi:10.1038/nrg2764-c2
Published online 18 November 2011

Thomas, D. Gene-environment-wide association studies: emerging approaches. Nature Rev. Genet. 11 259-272 (2010).

2. van IJzendoorn, M. H. et al. Gene-by-environment experiments: a new approach to finding the missing heritability. Nature Rev. Genet. 18 Nov 2011 (doi: 10.1038/nrg2764-c1)

3. Altshuler, D., Daly, M. J. \& Lander, E. S Genetic mapping in human disease. Science 322 881-888 (2008)

4. Gilliland, F. D., Li, Y. F., Saxon, A. \& Diaz-Sanchez, D. Effect of glutathione-S-transferase M1 and P1 genotypes on xenobiotic enhancement of allergic responses: randomised, placebo-controlled crossover study. Lancet 363, 119-125 (2004).

5. Li, R. Joint analysis of two related studies of different data types and different study designs using hierarchial modeling in detecting geneenvironment interactions. Thesis, Univ. Southern California (2011).

6. Islam, T. et al. Glutathione-S-transferase (GST) P1 GSTM1, exercise, ozone and asthma incidence in school children. Thorax 64, 197-202 (2009).

7. Bernstein, J. L. et al. Radiation exposure, the ATM gene, and contralateral breast cancer in the women's environmental cancer and radiation epidemiology study. J. Natl Cancer Inst. 102, 475-483 (2010)

8. Bernstein, J. L. et al. Study design: evaluating gene-environment interactions in the etiology of breast cancer - the WECARE study. Breast Cancer Res. 6, R199-R214 (2004).

9. Ioannidis, J. P. A. et al. A road map for efficient and reliable human genome epidemiology. Nature Genet. 38, 3-5 (2006).

Competing interests statement

The author declares no competing financial interests. 